\title{
Enantiomerization and Enantioselective Bioaccumulation of Metalaxyl in Tenebrio molitor Larvae
}

\author{
YONGXIN GAO, HUILI WANG, FANG QIN, PENG XU, XIAOTIAN LV, JIANZHONG LI, AND BAOYUAN GUO* \\ Research Center for Eco-Environmental Sciences, Chinese Academy of Sciences Haidian District, Beijing, China
}

\begin{abstract}
The enantiomerization and enantioselective bioaccumulation of metalaxyl by a single dose of exposure to Tenebrio molitor larvae under laboratory condition were studied by high-performance liquid chromatography tandem mass spectroscopy (HPLC-MS/MS) based on a ChiralcelOD-3R [cellulosetris-tris-(3, 5-dichlorophenyl-carbamate)] column. Exposure of enantiopure R-metalaxyl and S-metalaxyl in Tenebrio molitor larvae exhibited significant enantiomerization, with formation of the R enantiomers from the $\mathrm{S}$ enantiomers, and vice versa, which might be attributed to the chiral pesticide catalyzed by a certain enzyme in Tenebrio molitor larvae. Enantiomerization was not observed in wheat bran during the period of $21 \mathrm{~d}$. In addition, bioaccumulation of rac-metalaxyl in Tenebrio molitor larvae was enantioselective with a preferential accumulation of S-metalaxyl. These results showed that enantioselectivity was caused not only by actual degradation and metabolism but also by enantiomerization, which was an important process in the environmental fate and behavior of metalaxyl enantiomers. Chirality 26:88-94, 2014. 을 2013 Wiley Periodicals, Inc.
\end{abstract}

KEY WORDS: enantiomerization; enantioselectivity; bioaccumulation; metalaxyl; Tenebrio molitor larvae

\section{INTRODUCTION}

The most notorious environmental pollutants are pesticides, which are $\sim 40 \%$ chiral molecules. ${ }^{1-3}$ A chiral pesticide with one chiral center exists as two mirror image isomers called enantiomers possessing the same physical and chemical properties. The enantiomers of chiral pesticides may react at different rates with achiral molecules in the presence of chiral catalysts. Since constituents of living organisms are usually chiral, there are greater chances of the chiral pollutants to react at different rates, which may lead to variations in bioactivity, toxicity, biological uptake, metabolic pathways, degradation, and so on. ${ }^{4,5}$ Recently, a growing concern about the enantioselective behavior of chiral pesticides in the environment and the side effects of chiral pesticides on nontarget organisms have promoted the use of enantiomerically enriched or pure compounds as a means to diminish the amount of chemicals released into the environment. ${ }^{6,7}$ The enantio-specific profiles of chiral pesticides have become significant issues in the field of environmental chemistry and toxicology research.

Moreover, previous studies has suggested that some chiral pesticides were configurationally instable under certain conditions and might be easy to occur in enantiomerization, which was observed for mecoprop, dichlorprop, triadimefon, phenthoate, malathion, fenpropathrin, fenvalerate, cypermethrin, and so on. ${ }^{8-13}$ Enantiomerization of chiral pesticides could give rise to different influences on the environment. On the one hand, an active enantiomer could be converted to an inactive one with more toxicity and persistency; on the other hand, an inactive enantiomer might partly be converted into the active one. This could make the environmental behaviors and ecological effects of chiral pesticides complicated and unexpected. A detailed knowledge of the environmental behavior of biologically active chiral compounds must, therefore, contain extensive stereochemical information on enantiomeric stability. ${ }^{14}$

Metalaxyl [(R,S)-methyl-N-(2-methoxyacetyl)-N-(2,6-xylyl)DL-alaninate] (Fig. 1) is a chiral acylanilide fungicide widely (c) 2013 Wiley Periodicals, Inc. used in the control of plant diseases caused by pathogens of the Oomycora division in several crops. ${ }^{15,16}$ It was initially marketed in the form of a racemate containing two enantiomers, $\mathrm{R}$ and $\mathrm{S}$, although its fungicidal activity mostly originates from the R-enantiomer. At present, R-metalaxyl has been used in place of $r a c$-metalaxyl in many countries. ${ }^{17,18}$ To date, a number of investigations have reported metalaxyl enantioselective behavior in a variety of ecosystems ${ }^{19-22}$ and animals, ${ }^{23,24}$ and the results indicated that both environmental media and conditions could greatly influence the direction and rate of enantioselective degradation and transformation of the metalaxyl enantiomers. However, to our knowledge, except for the study of enantiomerization of metalaxyl in soil ${ }^{20}$ and sunflower plants, ${ }^{25}$ data concerning the enantiomerization and enantioselective bioaccumulation of metalaxyl in other nontarget organisms and especially in insects are limited, although this pesticide is widely applied. (Figure 2)

As is known, insects are the most diverse groups of animals distributed in nature, which are largely responsible for the breakdown of organic materials such as plants, animals, and human remains and the elimination of animal wastes. They are essential food sources for many birds, fishes, reptiles, and amphibians and they also constitute a significant part of the human diet in some parts of the world. ${ }^{26,27}$ With a large number of pesticides widely abused in China, insects can be infected through touching the agricultural products contaminated by the various insecticides, herbicides, or fungicides and transferred to the higher trophic level of predatory insects. Tenebrio molitor larvae are a kind of lepidoptera insects in stored grain products and are often used as a model

\footnotetext{
*Correspondence to: Baoyuan Guo, Research Center for Eco-Environmental Sciences, Chinese Academy of Sciences, Shuangqing RD 18, Haidian District, Beijing 100085, China. E-mail: guoby@rcees.ac.cn

Received for publication 25 July 2013; Accepted 13 October 2013

DOI: 10.1002/chir.22269

Published online 3 December 2013 in Wiley Online Library

(wileyonlinelibrary.com).
} 


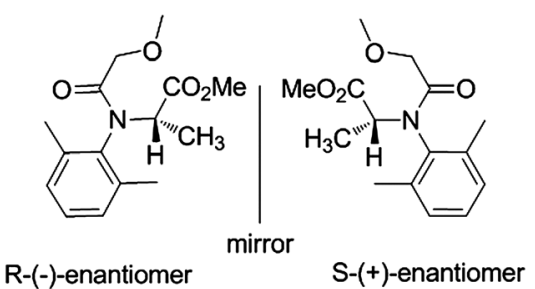

Fig. 1. Structures of furalaxyl enantiomers.

species in the fields of environmental toxicology, genetics, and so on. ${ }^{28,29}$ So far, no research has yet been reported for the enantiomerization and enantioselective bioaccumulation of the metalaxyl enantiomers in Tenebrio molitor larvae.

In this article, an effective method for the stereoselective determination of metalaxyl residues in Tenebrio molitor larvae and wheat bran was developed by high-performance liquid chromatography tandem mass spectroscopy (HPLC-MS/ MS). Enantiopure metalaxyl enantiomers were employed to elucidate potential enantiomerization of metalaxyl in Tenebrio molitor larvae. In addition, enantioselective bioaccumulation of metalaxyl in Tenebrio molitor larvae was investigated. The purpose of the present study is to emphasize the importance of enantiomerization in the environmental fate and behavior of chiral pesticides and to accurately evaluate the potential effects of the metalaxyl enantiomers on organisms.

\section{MATERIAL AND METHODS Chemicals and Reagents}

The fungicide of $r a c$-metalaxyl ( $>98.0 \%$ purity) was provided by the China Ministry of Agriculture Institute for Control of Agrochemicals. Two metalaxyl enantiomers were prepared via chiral synthesis methods, and the optical purities of the R-enantiomer and S-enantiomer were both about $97.0 \%$. Water was purified by a Milli-Q system. Methanol (HPLC grade), n-Hexane (HPLC grade), and acetonitrile (HPLC grade) were obtained from Dikma (Lake Forest, CA). Pyridine, trifluoromethanesulfonic anhydride, methyl (S)-(-)-lactate, methyl (R)-(+)-lactate, 2, 6-dimethyl aniline, 2-methoxyacetyl chloride, sodium bicarbonate, toluene, dichloromethane $\left(\mathrm{CH}_{2} \mathrm{Cl}_{2}\right)$, ether, ethyl acetate, acetone, acetonitrile (analytical grade), and anhydrous sodium sulfate $\left(\mathrm{Na}_{2} \mathrm{SO}_{4}\right)$ were purchased from Beijing Chemical Reagent (Beijing, China).

\section{HPLC-MS/MS Conditions}

HPLC was performed using Thermo ACCELA series (Thermo Electron, Hopkinson, MA) equipped with ACCELA Autosampler, ACCELA
600 pump, a $20 \mu \mathrm{L}$ and a $2 \mu \mathrm{L}$ flow cell. Enantiomers were separated on a ChiralcelOD-3R [cellulosetris-tris-(3, 5-dichlorophenyl-carbamate)] column. The mobile phase was a mixture of $75 \%$ acetonitrile and $25 \%$ water with a flow rate of $0.4 \mathrm{~mL} / \mathrm{min}$. Chromatographic separation was conducted at $20^{\circ} \mathrm{C}$ and the injection volume was $1 \mu \mathrm{L}$.

TSQ QUANTUM ACCESS MAX was used for LC-MS/MS analysis (Thermo Electron). Quantification was achieved in positive-ion mode (ESI+) with selected reaction monitoring (SRM). The signals were received and processed with Thermo Xcalibur 2.2 SP1.48 software. The optimized major working parameters were as follows: Spray Voltage $3200 \mathrm{~V}$, Vaporizer Temperature $250^{\circ} \mathrm{C}$, Sheath Gas Pressure $30 \mathrm{psi}$, Aux Gas Pressure 10 arbitrary units, Capillary Temperature $350^{\circ} \mathrm{C}$, Capillary Offset $35 \mathrm{~V}$, and Q2 Gas Pressure 1.5 psi. For metalaxyl, transition $\mathrm{m} / \mathrm{z}$ $280>220$ was used for confirmation, $\mathrm{m} / \mathrm{z} 280>160$ was used for quantification, and collision energies were $7 \mathrm{eV}$ and $18 \mathrm{eV}$, respectively.

\section{Synthesis of the Two Enantiomers of Metalaxyl}

To better explore the enantiomerization of chiral pesticides in organisms, obtaining sufficient enantiopure enantiomers standards is necessary. In the study, the two metalaxyl enantiomers were prepared via chiral synthesis methods.

\section{General Procedure 30,31}

Synthesis of N-xylyl-D-methyl alaninate. Pyridine $(1.42 \mathrm{~mL}, 17.6 \mathrm{mmol}$, 1.1 equiv) in $30 \mathrm{~mL}$ of $\mathrm{CH}_{2} \mathrm{Cl}_{2}$ was cooled to $0^{\circ} \mathrm{C}$, trifluoromethanesulfonic anhydride (2.8 $\mathrm{mL}, 16.2 \mathrm{mmol}, 1.05$ equiv) was added, and the resulting mixture was stirred for $10 \mathrm{~min}$. Methyl (S)-(-)-lactate $(1.53 \mathrm{~mL}, 16.0 \mathrm{mmol}$, 1 equiv) was added dropwise. The reaction mixture was stirred at $0^{\circ} \mathrm{C}$ for $30 \mathrm{~min}$ and then warmed to room temperature. The mixture was filtered and the filter cake was washed with ether. The filtrate was evaporated in vacuo $\left(<25^{\circ} \mathrm{C}\right)$, and the residue was triturated with ether and filtered. After evaporating the solvent, the residue was dissolved in $20 \mathrm{~mL}$ of dichloromethane and 2, 6-dimethyl aniline (1.97 mL, $16.0 \mathrm{mmol}, 1$ equiv) was added. The reaction solution was stirred at room temperature overnight. Removal of the solvent in vacuo followed by flash chromatography gave a light yellow oil $(2.76 \mathrm{~g})$.

Synthesis of R-metalaxyl. Sodium bicarbonate ( $0.5 \mathrm{~g}, 6 \mathrm{mmol}, 1.1$ equiv) were added to a solution of N-xylyl-D-methyl alaninate $(1.12 \mathrm{~g}, 5.5 \mathrm{mmol}$, 1 equiv) in $30 \mathrm{~mL}$ of toluene, cooled to a temperature ranging from $5^{\circ} \mathrm{C}$ $10^{\circ} \mathrm{C}$, and subsequently 2 -furoyl chloride $(0.65 \mathrm{~g}, 6 \mathrm{mmol}, 1.1$ equiv) was slowly added dropwise. After $4 \mathrm{~h}$ at room temperature, the above solution was washed with saturated sodium chloride and the organic phase was evaporated in vacuo. The obtained crude product was crystallized with hexane to give $1.2 \mathrm{~g}$ of white crystalline solid alaninate with an enantiomeric $\mathrm{R} / \mathrm{S}$ ratio $=97$ (yield $68 \%$ ).

Synthesis of S-metalaxyl with methyl (R)-(+)-lactate as a starting material followed the same experimental procedure.
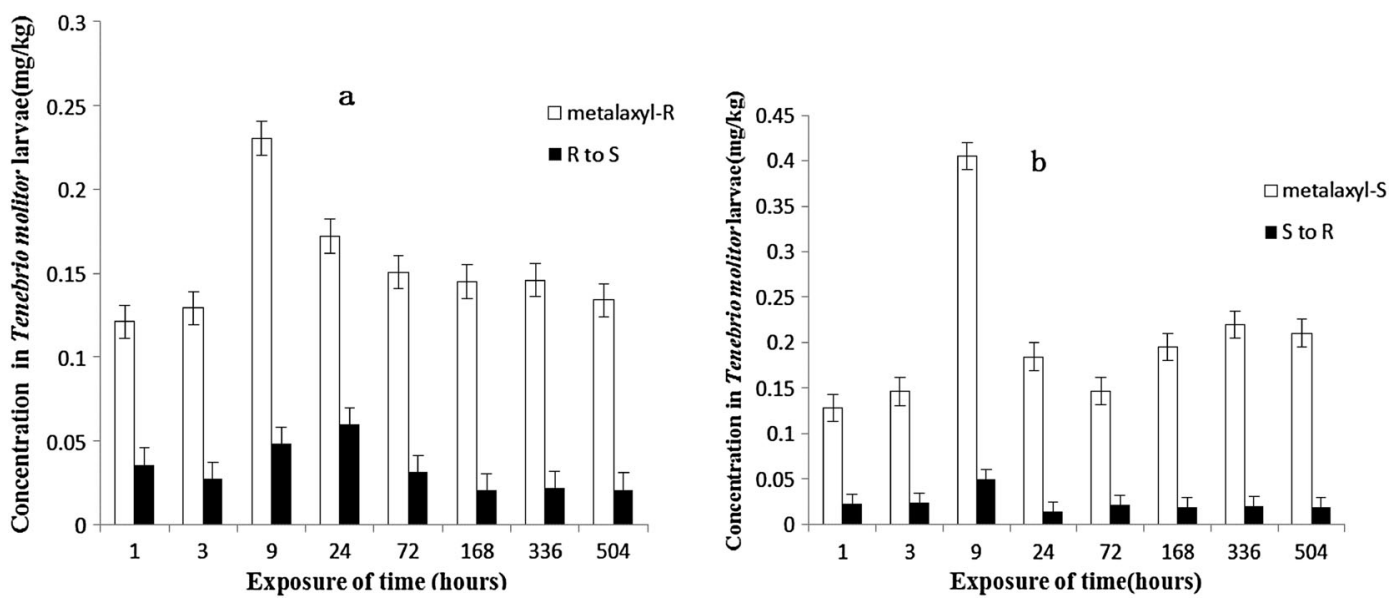

Fig. 2. Enantiomerization of enantiopure R-metalaxyl (a) and enantiopure S-metalaxyl (b) in Tenebrio molitor larvae. 


\section{Tenebrio molitor Larvae}

Tenebrio molitor larvae were purchased from Beijing jie matt biological technology (Beijing, China) and were reared in ventilated plastic terrariums $(25 \times 15 \times 20 \mathrm{~cm})$. A layer of wheat bran covered the terrarium floor. The population was kept at $25^{\circ} \mathrm{C}$ and the light cycle adopted was $16 \mathrm{~h}$ light and $8 \mathrm{~h}$ dark.

\section{Wheat Bran Formulation and Tenebrio molitor Larvae Exposure}

To ensure that the rac-metalaxyl was dispersed uniformly in the $100 \mathrm{~g}$ dry weight of wheat bran, we did the procedure in steps (dilution spike). First, $r a c$-metalaxyl was dissolved in acetonitrile to make a stock solution at a concentration of $1000 \mathrm{mg} / \mathrm{L}$. Then $1 \mathrm{~mL}$ of acetonitrile solution $(1000$ $\mathrm{mg} / \mathrm{L}$ ) was dissolved into $50 \mathrm{~mL}$ of acetone; meanwhile, $100 \mathrm{~g}$ of wheat bran was soaked in the $50 \mathrm{~mL}$ of acetone solution of rac-metalaxyl, followed by drying in a fuming cupboard overnight. The wheat bran contaminated with enantiopure R-metalaxyl and S-metalaxyl followed the same experimental procedure.

In this experiment, Tenebrio molitor was used in the larval stage $(0.1 \mathrm{~g}$ mean body weight). Molting or pupating larvae were excluded from the experiment. Before the worms were introduced, they were fed with uncontaminated wheat bran for 1 week to acclimate. A piece of paper resting on top of the bran provided cover for the worms. $30 \mathrm{~g}$ wet weight of Tenebrio molitor larvae were exposed to the chemical in each ventilated plastic terrarium containing $7 \mathrm{~g}$ wet weight of contaminated wheat bran. Food was renewed once a week. All of the plastic terrariums were placed in the dark in an environmental chamber.

For the uptake experiment, $1 \mathrm{~g}$ of Tenebrio molitor larvae and $0.5 \mathrm{~g}$ of wheat bran were collected after an exposure period $(1,3,9,24,72,168$, 336 , and $504 \mathrm{~h}$ ). Wheat bran on the surface of worms was filtered by a sieve, and the worms were frozen at $-20^{\circ} \mathrm{C}$ in $50 \mathrm{~mL}$ of a polypropylene centrifuge tube. Wheat bran samples from each plastic terrarium were also stored at $-20^{\circ} \mathrm{C}$. All experiments were performed at $25 \pm 0.5^{\circ} \mathrm{C}$ and were run in triplicate at each sample point.

\section{Analysis of Metalaxyl Residue}

For the sample pretreatment of wheat bran, $15 \mathrm{~mL}$ of acetonitrile was added to a $50 \mathrm{~mL}$ polypropylene centrifuge tube containing $0.5 \mathrm{~g}$ wet weight of incubated wheat bran sample. Next, the tube was stirred for 3 min on a vortex mixer, exposed to ultrasonic vibration for $10 \mathrm{~min}$, and then centrifuged at $3000 \mathrm{rpm}$ for $5 \mathrm{~min}$. The extract was transferred to a new tube. The remaining part was extracted again following the same extraction step, and the extract was combined. The combined extract was filtered by a funnel with $5 \mathrm{~g}$ of anhydrous sodium sulfate to a pear-shaped flask and evaporated to dryness by a vacuum rotary evaporator at $35^{\circ} \mathrm{C}$. The alumina-N-solid-phase extraction (SPE) $(1.0 \mathrm{~g})$ on a cartridge $(6 \mathrm{~mL})$ was used to clean up other interfering substances. The cartridge was preconditioned by rinsing with $5 \mathrm{~mL}$ of ethyl acetate followed by $5 \mathrm{~mL}$ of $\mathrm{n}$-hexane and equilibrated with $5 \mathrm{~mL}$ of 1:2 ethyl acetate:n-hexane. The sample of dry extract was dissolved in $2 \mathrm{~mL}$ of $20 \%$ ethyl acetate in n-hexane, then the solution was passed through the SPE cartridge. The cartridge was eluted with additional $8 \mathrm{~mL}$ of $1: 2$ ethyl acetate: $n$-hexane. The eluates were combined with the loading eluates. The combined $10 \mathrm{~mL}$ of eluate was collected in a pear-shaped flask and evaporated to dryness by a vacuum rotary evaporator at $35^{\circ} \mathrm{C}$ and the dry extract was diluted to $1.0 \mathrm{~mL}$ with acetonitrile (HPLC grade) and passed through a filter membrane (pore size, $0.22 \mu \mathrm{m})$.

For analysis of the Tenebrio molitor larvae, the samples were thawed for about $15 \mathrm{~min}$ at room temperature. $15 \mathrm{~mL}$ of acetonitrile was added to each tube containing $1 \mathrm{~g}$ wet weight of Tenebrio molitor larvae sample. Next, the tube was stirred for $3 \mathrm{~min}$ on a vortex mixer, exposed to ultrasonic vibration for $10 \mathrm{~min}$, and then centrifuged at $3000 \mathrm{rpm}$ for $5 \mathrm{~min}$. The sample was reextracted in the same way and the supernatants were combined. The combined extract was filtered by a funnel with about $5 \mathrm{~g}$ of anhydrous sodium sulfate to a pear-shaped flask and evaporated to dryness at $35^{\circ} \mathrm{C}$. For cleanup (fat destruction), $3 \times 10 \mathrm{~mL}$ of n-hexane was added for liquid-liquid partition to extract most of the lipid. The upper layer of n-hexane was discarded, and the layer of acetonitrile was Chirality DOI 10.1002/chir evaporated to dryness by a vacuum rotary evaporator. The alumina-Nsolid-phase extraction (SPE) $(1.0 \mathrm{~g})$ on a cartridge $(6 \mathrm{~mL})$ was used to clean up other interfering substances. The cartridge was preconditioned by rinsing with $5 \mathrm{~mL}$ of ethyl acetate followed by $5 \mathrm{~mL}$ of $\mathrm{n}$-hexane and equilibrated with $10 \mathrm{~mL}$ of 1:2 ethyl acetate:n-hexane. The sample of dry extract was dissolved in $2 \mathrm{~mL}$ of $20 \%$ ethyl acetate in n-hexane, then the solution was passed through the SPE cartridge. The cartridge was eluted with an additional $8 \mathrm{~mL}$ of $1: 2$ ethyl acetate:n-hexane. The eluates were combined with the loading eluates. The combined $10 \mathrm{~mL}$ of eluate was collected in a pear-shaped flask and evaporated to dryness by a vacuum rotary evaporator at $35^{\circ} \mathrm{C}$ and the dry extract was diluted to $1.0 \mathrm{~mL}$ with acetonitrile (HPLC grade) and passed through a filter membrane (pore size, $0.22 \mu \mathrm{m}$ ).

\section{Method and Validation}

A stock solution $(100 \mathrm{mg} / \mathrm{L})$ of $r a c$-metalaxyl and two enantiomers were prepared in acetonitrile,. A series of standard working solutions at $10,50,100,500$, and $1000 \mu \mathrm{g} / \mathrm{L}$ concentrations were prepared from the stock solution by serial dilution in acetonitrile. According to the procedure described above, matrix-matched standard solutions were obtained at the same concentrations by adding blank Tenebrio molitor larvae and wheat bran sample extracts to each serially diluted standard solution. Calibration curves were generated by plotting the peak area of quantification ion transition against the concentrations of the enantiomers with regression analysis.

Blank samples (Tenebrio molitor larvae and wheat bran) analysis was performed to check interference from the matrix. The slope ratios of the linear calibration functions were calculated to differentiate between the extraction efficiency and the matrix-induced signal suppression/ enhancement (SSE). The SSE caused by matrix effects was determined.

Recovery of metalaxyl enantiomers was measured in a blank sample that was fortified at different levels $(0.1,0.5$, and $1 \mathrm{mg} / \mathrm{L}$ based on five replicates). The samples were left for $1 \mathrm{~h}$ to ensure that the spiked samples were evenly distributed. The fortified samples were analyzed and the recoveries were calculated by comparing the measured concentration to the fortified concentrations. The precision in the condition for repeatability, expressed as the relative standard deviation (RSD), was determined at the entire calibration range.

The stability of the metalaxyl enantiomers were evaluated in the stock solution and matrix extracts. The stock solutions of the enantiomers, which were prepared two months previously, were tested by comparison of injections of a newly prepared working solution. The stability of the spiked Tenebrio molitor larvae and wheat bran samples for the enantiomers were determined, and all the samples used in the stability test were stored at $-20^{\circ} \mathrm{C}$.

\section{RESULTS AND DISCUSSION Calibration and Method Validation}

The chiral LC-MS/MS method described above was validated for the analysis of metalaxyl in Tenebrio molitor larvae and wheat bran. The typical chromatogram of R-metalaxyl and S-metalaxyl was baseline separated with no endogenous interference peaks (Fig. 3). Good linearity was obtained over the concentration range $0.01-1 \mathrm{mg} / \mathrm{kg}$, and the correlation coefficients $\left(\mathrm{r}^{2}\right)$ of R-metalaxyl and S-metalaxyl were 0.9961 and 0.9968 , and the equations of the linear regression are $y=9.0 \times 10^{6} x+268332$ and $y=9.0 \times 10^{6} x+255584$, respectively. If the concentrations of the analytical extract exceed the linear range of the analysis, the analytical extract should be diluted or concentrated. Tenebrio molitor larvae samples were fortified with $0.1,0.5$, and $1 \mathrm{mg} / \mathrm{kg}$ of the metalaxyl enantiomers. The matrix effect was calculated by comparing the slope of matrix-matched standard curve with the slope of the standard calibration curve, and the slope ratios were in the range of $0.85-1.14$, so there was no significant matrix effect for metalaxyl determination with HPLC-MS/MS based 

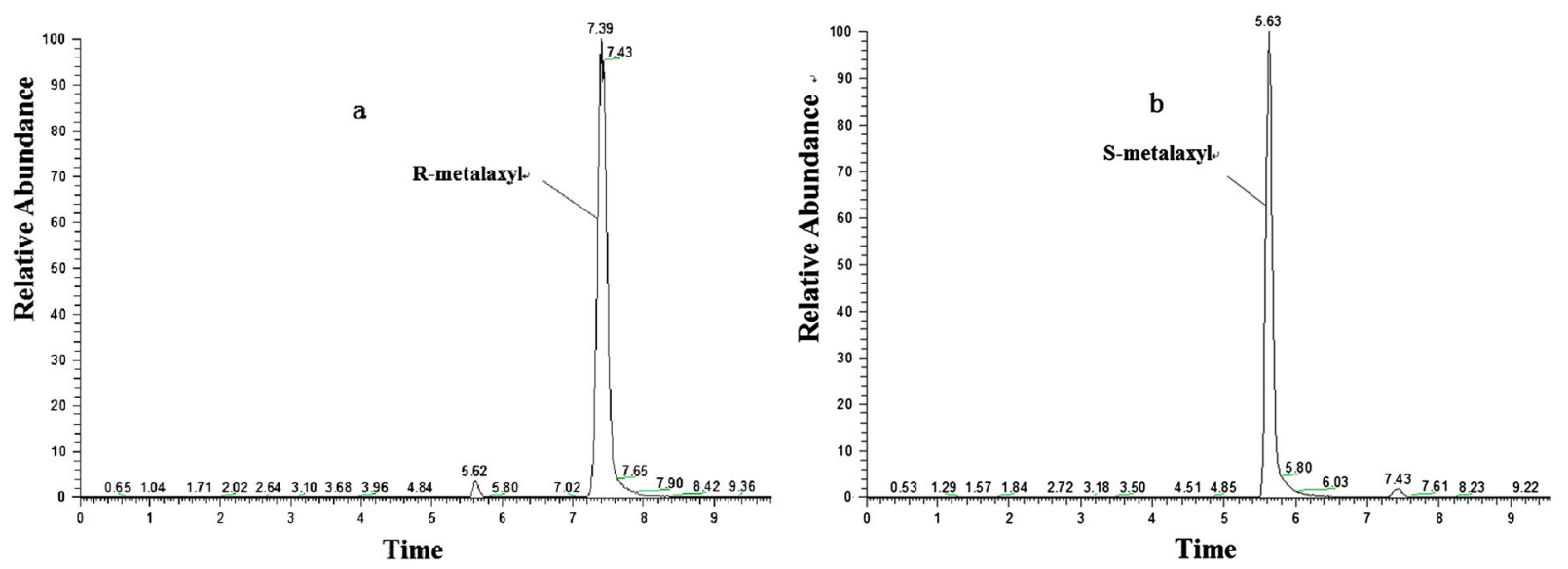

Fig. 3. Representative HPLC-MS chromatogram of the expections of rac-metalaxyl enantiomers in Tenebrio molitor larvae after 21 days of exposure. Flow rate $=0.4 \mathrm{~mL} / \mathrm{min}$, ultrapure water: acetonitrile $=25: 75(V / V)$.

on the proposed method. The average recoveries for both enantiomers ranged between $86 \%$ and $94 \%$ in Tenebrio molitor larvae and between $90 \%$ and $95 \%$ in wheat bran, and the RSDs ranged from $2.8 \%-7.9 \%$ in Tenebrio molitor larvae, and $2.5 \%$ to $5.6 \%$ in wheat bran. Based on the peak-to-peak signal-to-noise ratio of 3 , the limit of detection of the method was $0.02 \mathrm{mg} / \mathrm{kg}$ both in Tenebrio molitor larvae and wheat bran.

\section{Enantiomerization in Tenebrio molitor Larvae}

To accurately investigate enantiomerization of metalaxyl enantiomers in Tenebrio molitor larvae, enantiomerization was evaluated first in wheat bran. Figure 4 shows that no enantiomerization occurred for the metalaxyl enantiomers in wheat bran during $21 \mathrm{~d}$, demonstrating that metalaxyl enantiomers were configurationally stable in wheat bran. Then the wheat bran contaminated with enantiopure R-metalaxyl and S-metalaxyl was fed to Tenebrio molitor larvae,. The results after exposure for $21 \mathrm{~d}$ are shown in Figure 4a,b. With respect to enantiopure R-metalaxyl, the concentration of R-metalaxyl rapidly increased to $0.23 \mathrm{mg} / \mathrm{kg}$ in $9 \mathrm{~h}$, and then quickly decreased and reached a steady-state level in 3-21 d. Besides, obvious enantiomerization from $\mathrm{R}$ to $\mathrm{S}$ was observed at each point of exposure time. Concentration of S-metalaxyl reached up to $0.06 \mathrm{mg} / \mathrm{kg}$ in 24 hours, and then the degree of conversion gradually became weak and reached a steady-state level.

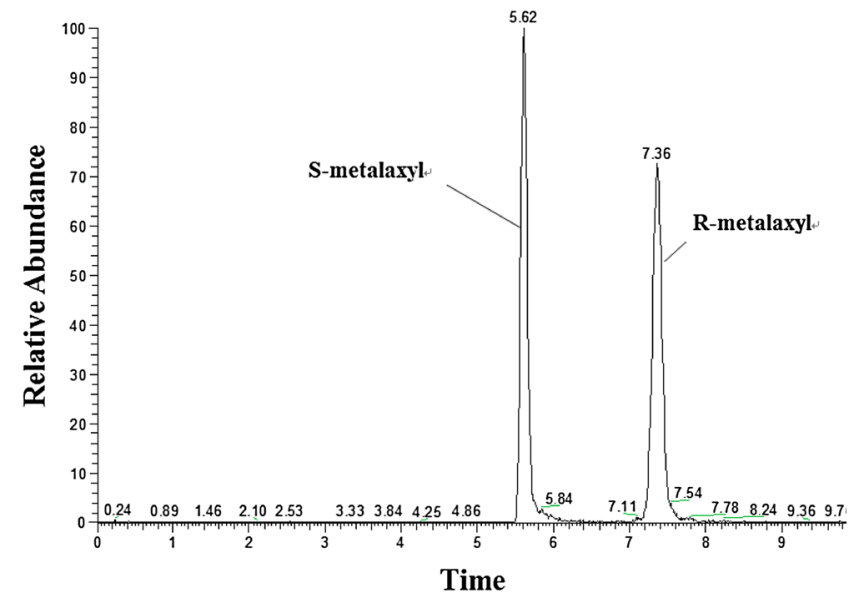

Fig. 4. Representative HPLC-MS chromatogram of the expections of (a) enantiopure R-metalaxyl in wheat bran and (b) enantiopure S-metalaxyl in wheat bran after $21 \mathrm{~d}$ of exposure. Flow rate $=0.4 \mathrm{~mL} / \mathrm{min}$, ultrapure water: acetonitrile $=25: 75(V / V)$.
With respect to enantiopure S-metalaxyl, the enantiomerization from $\mathrm{S}$ to $\mathrm{R}$ in Tenebrio molitor larvae was also observed and the change trend of the concentration was similar to that of the enantiopure R-metalaxyl. According to the above results, apparently the metalaxyl enantiomers underwent enantiomerization at a fast rate in Tenebrio molitor larvae. It was not surprising that the biotransformation process of chiral pesticides could lead to an inversion of configuration, but so a fast conversion rate in Tenebrio molitor larvae exceeded our expectations. We considered that the rapid enantiomerization of metalaxyl enantiomers exhibited a strong excitability for Tenebrio molitor larvae. At the later phase of exposure, the extent of enantiomerization got smaller, due to continuous uptake of the major enantiomer and degradation of the minor enantiomer. Although the rate of enantiomerization of enantiomer R-metalaxyl and S-metalaxyl could not be directly calculated, it seemed that the rate of enantiomerization ( $\mathrm{R}$ to $\mathrm{S}$ ) was different from that of enantiomerization ( $\mathrm{S}$ to $\mathrm{R}$ ) based on the degree of conversion. It has been reported that in chemically mediated reactions, both enantiomers of a chiral compound interconvert, and the rates of enantiomerization ( $R$ to $S$ ) and ( $\mathrm{S}$ to $\mathrm{R}$ ) are equal, ${ }^{8}$ and in enzyme-catalyzed reactions, these rates generally differ, and the formation of one enantiomer over the other is favored. ${ }^{32}$ Therefore, to some extent, the results of the experiment illustrated enantiomerization could be attributed to enzyme-catalyzed reactions with enantiomers of metalalxyl in Tenebrio molitor larvae.

Enzyme-mediated enantiomerization had been extensively studied for pharmaceuticals, ${ }^{33,34}$ amino acids, and sugars ${ }^{35}$ with respect to its mechanism. A plausible explanation of the relationship between enantiomerization and chemical structure was given. The inversion of stereochemistry in a chiral compound was generally catalyzed by racemases or epimerases through cleavage of the methane $\mathrm{C}-\mathrm{H}$ bond adjacent to a carbonyl group followed by its reformation or successive oxidation-reduction at a chiral center. ${ }^{36}$ As seen in Figure 1, the chiral carbon in metalaxyl holds an electronwithdrawing carbonyl group and a conjugated acetanilide functional group. In the light of the principle of structural chemistry, the proton on the chiral center is relatively acidic and is thus easily lost to create an intermediate carbanion. Under protic environment, reprotonation regenerates the parent compound from either the top or the bottom face of the anion, thus interconverts enantiomers. 
We believed that there should be more chiral pesticides undergoing such enantiomerization that may occur in a chiral environment and this deserves further investigation. This was particularly important with more and more enantiopure products being introduced to the market.

\section{Enantioselective Bioaccumulation in Tenebrio molitor Larvae}

Enantioselective bioaccumulation in Tenebrio molitor larvae was investigated with rac-metalaxyl. The concentrations of the two metalaxyl enantiomers in Tenebrio molitor larvae were determined. The result of bioaccumulation of Tenebrio molitor larvae is shown in Figure 5. The rapid uptake of the two metalaxyl enantiomers was observed in Tenebrio molitor larvae at the initial phase. Concentrations of the two enantiomers both reached the highest level in $9 \mathrm{~h}$. But the rapid decrease of concentrations was observed between 9 to $24 \mathrm{~h}$ of exposure. Finally, the concentrations tended to stability after 3 d. In Figure 5, a significant difference was observed between the concentrations of two enantiomers in Tenebrio molitor larvae tissue at all sample points, with the concentration of S-metalaxyl higher than that of R-metalaxyl. Another article has reported that the concentration of S-metalaxyl was higher than that of R-metalaxyl from soil to earthworms, ${ }^{24}$ the result of which is in accordance with that of the experiments.

The enantiomer fraction (EF) was employed to measure the enantioselectivity of the bioaccumulation of enantiomers of benalaxyl in Tenebrio molitor larvae. The EF values defined range from 0 to 1 , with $\mathrm{EF}=0.5$ representing the racemic mixture. $\mathrm{EF}$ was expressed as follows:

$$
\mathrm{EF}=[\mathrm{S}] /[\mathrm{R}]+[\mathrm{S}]
$$

The EF values calculated in Tenebrio molitor larvae tissue are shown in Figure 6. It was observed that the EF values deviated from 0.5 in the bioaccumulation experiment during $21 \mathrm{~d}$ of exposure, reached the highest level in $9 \mathrm{~h}$, and finally maintained at $\sim 0.58$. A paired $t$ test for the same timepoints between R-metalaxyl and S-metalaxyl yielded a $P$ value of 0.002 . The paired $t$ test exhibited a significant difference between the concentrations of two enantiomers in Tenebrio molitor larvae during the course of the experiment. The change of $\mathrm{EF}$ indicated an initial predominance of S-enantiomer in comparison to R-enantiomer, but with time the S-enantiomer

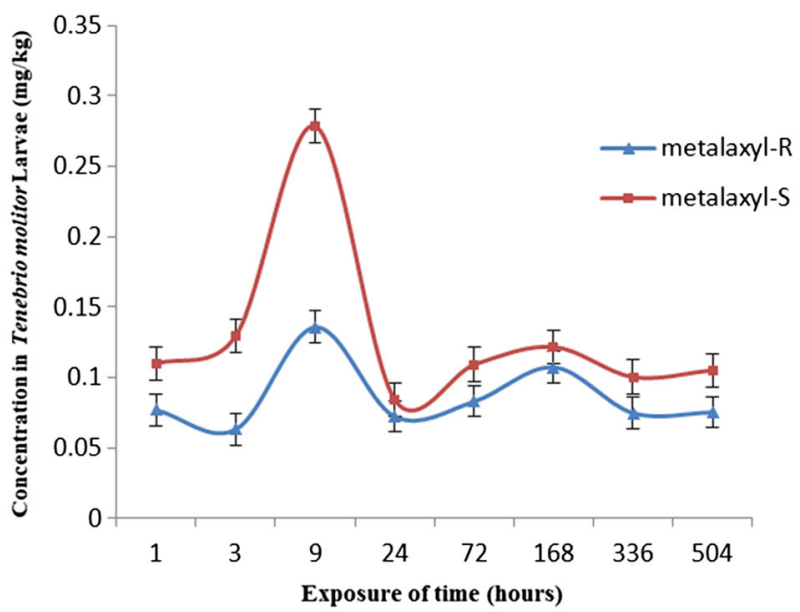

Fig. 5. Concentrations of rac-metalaxyl enantiomers in Tenebrio molitor larvae.

Chirality DOI 10.1002/chir

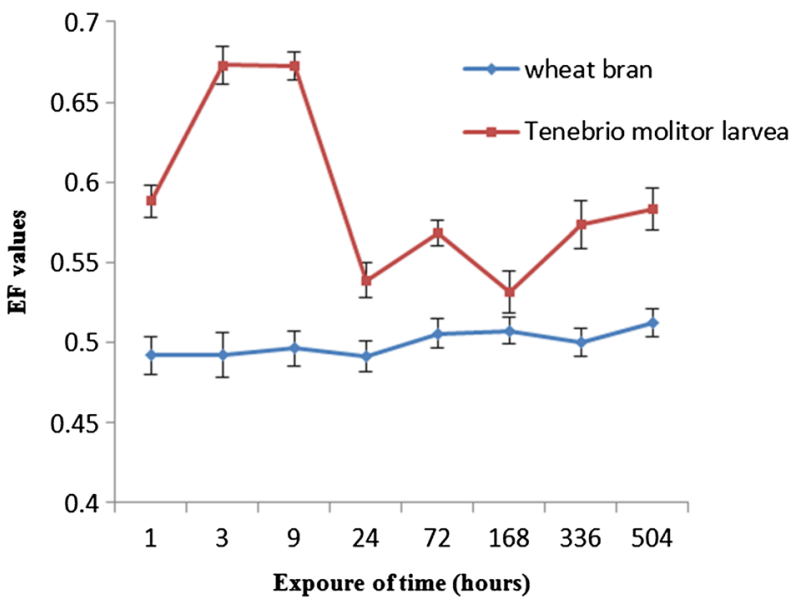

Fig. 6. Enantiomeric fraction (EF) of rac-metalaxyl residue in Tenebrio molitor larvae and wheat bran.

disappeared faster than the R-enantiomer, so the bioaccumulation of metalaxyl in Tenebrio molitor larvae for this treatment was expected to be an enantioselective process. The result was in accordance with what the earlier paper reported on enantioselective bioaccumulation of metalaxyl in earthworm. ${ }^{24}$

In this work, the bioaccumulation factor (BAF) is used to express the bioaccumulation of the metalaxyl enantiomers. $\mathrm{BAF}$ is a function of the relative sorptive capacities of organism versus the surrounding food, and is defined as:

$$
\mathrm{BAF}=\mathrm{C}_{\mathrm{T}} / \mathrm{C}_{\mathrm{WB}}
$$

where $\mathrm{C}_{\mathrm{T}}$ is the concentration of compound in Tenebrio molitor larvae tissue and $\mathrm{C}_{\mathrm{WB}}$ is the concentration of compound in wheat bran. Since the experiment was confined to one species, the BAF was not normalized to the lipid content of the organism. ${ }^{37}$ Steady state was defined as the time only when concentrations did not continue to obviously increase or decrease over three consecutive sampling intervals. ${ }^{38}$ The calculated BAF was 0.02 for R-metalaxyl and 0.015 for S-metalaxyl in the steady state. A paired $t$ test for the steady state between R-metalaxyl and S-metalaxyl yielded a $P$ value of 0.013 , showing that there was a significant difference in $\mathrm{BAF}$ between the two enantiomers of metalaxyl. The BAF value of the S-enantiomer was higher than that of the R-enantiomer, which revealed that the S-enantiomer was preferentially accumulated over the R-enantiomer in Tenebrio molitor larvae. It could be assumed that the bioaccumulation was enantioselective.

From the results of the experiments, the metalaxyl enantiomers exhibited a low capacity of bioaccumulation in Tenebrio molitor larvae and the characteristics observed were that the metalaxyl enantiomers were taken up rapidly and the concentrations of metalaxyl reached the highest level in a short time and then decreased quickly until equilibration. The low capacity of bioaccumulation of metalaxyl could be attributed to a slow uptake rate by Tenebrio molitor larvae, the low lipophilicity of metalaxyl, and a high degradation rate.

As obvious enantiomerization of R-metalaxyl and S-metalaxyl was observed in Tenebrio molitor larvae before, enantiomerization should be considered one of the possible factors which brought about enantioselective bioaccumulation of metalaxyl in Tenebrio molitor larvae. This showed that the enantioselective behavior of chiral pesticides in organisms was the result of a combination of enantioselective degradation and enantiomerization. The use of each enantiomer as a starting material can clearly 
demonstrate the involvement of an enantiomerization process by the appearance of the corresponding enantiomer and correctly evaluating the enantioselectivity and enantiomerization of chiral pesticides. Hence, the behavior of each enantiomer in ecotoxicology on the most sensitive species should be ideally examined with enantiomerization to more correctly assess the safety of a chiral pesticide.

\section{CONCLUSION}

In our experiment, the enantiomerization and enantioselective bioaccumulation of the metalaxyl enantiomers in Tenebrio molitor larvae from wheat bran were investigated by HPLCMS/MS based on a ChiralcelOD-3R column. The results indicated that exposure of enantiopure R-benalaxyl and S-benalaxyl in Tenebrio molitor larvae revealed significant enantiomerization with formation of the $\mathrm{R}$ enantiomers from the $\mathrm{S}$ enantiomers, and vice versa; enantiomerization was not observed in wheat bran during the period of $21 \mathrm{~d}$. In addition, there was an obvious enantioselective bioaccumulation in the larvae with a preferential accumulation of S-metalaxyl at $10 \mathrm{mg} / \mathrm{kg}^{-1}$ exposure. Much more attention should be paid to enantiomerization for a better understanding of the chiral profiles of relevant chiral pesticides in the environment.

\section{ACKNOWLEDGMENTS}

This work has been funded by the Innovative Program of the Chinese Academy of Sciences (KZCX2-YW-JS403) and National High Technology Research and Development Program (863) of China (No. 2012AA06A302).

\section{SUPPORTING INFORMATION}

Additional supporting information may be found in the online version of this article at the publisher's web-site.

\section{LITERATURE CITED}

1. Lewis DL, Garrison AW, Wommack KE, Whittemore A, Steudler P, Melillo J. Influence of environmental changes on degradation of chiral pollutants in soils. Nature 1999;401:898-901.

2. Garrison AW. Probing the enantioselectivity of chiral pesticides. Environ Sci Technol 2006;40:16-23.

3. Ye J, Zhao M, Liu J, Liu W. Enantioselectivity in environmental risk assessment of modern chiral pesticides. Environ Pollut 2010;158:2371-2383.

4. Buser HR, Mueller MD, Rappe C. Enantioselective determination of chlordane components using chiral high-resolution gas chromatographymass spectrometry with application to environmental samples. Environ Sci Technol 1992;26:1533-1540.

5. Maier NM, Franco P, Lindner W. Separation of enantiomers: needs, challenges, perspectives. J Chromatog A 2001;906:3-33.

6. Buser H-R, Müller MD. Occurrence and transformation reactions of chiral and achiral phenoxyalkanoic acid herbicides in lakes and rivers in Switzerland. Environ Sci Technol 1998;32:626-633.

7. Buser H-R, Poiger T, Müller MD. Changed enantiomer composition of metolachlor in surface water following the introduction of the enantiomerically enriched product to the market. Environ Sci Technol 2000;34:2690-2696.

8. Müller MD, Buser H-R. Conversion reactions of various phenoxyalkanoic acid herbicides in soil. 1. Enantiomerization and enantioselective degradation of the chiral 2-phenoxypropionic acid herbicides. Environ Sci Technol 1997;31:1953-1959.

9. Li Z, Wu T, Li Q, Zhang B, Wang W, Li J. Characterization of racemization of chiral pesticides in organic solvents and water. J Chromatog A 2010;1217:5718-5723.

10. Nillos MG, Qin S, Larive C, Schlenk D, Gan J. Epimerization of cypermethrin stereoisomers in alcohols. J Aagric Food Chem 2009;57:6938-6943.
11. Liu W, Qin S, Gan J. Chiral stability of synthetic pyrethroid insecticides. J Agric Food Chem 2005;53:3814-3820.

12. Qin S, Gan J. Abiotic enantiomerization of permethrin and cypermethrin: Effects of organic solvents. J Agric Food Chem 2007;55:5734-5739.

13. Li Z, Zhang Y, Li Q, Wang W, Li J. Enantioselective degradation, abiotic racemization, and chiral transformation of triadimefon in soils. Environ Sci Technol 2011;45:2797-2803.

14. Cannazza G, Battisti U, Carrozzo MM, Brasili L, Braghiroli D, Parenti C. Evaluation of stereo and chemical stability of chiral compounds. Chirality 2011;23:851.

15. Fisher DJ, Hayes AL. Mode of action of the systemic fungicides furalaxyl, metalaxyl and ofurace. Pest Sci 1982;13:330-339.

16. Mueller MD, Buser H-R. Environmental behavior of acetamide pesticide stereoisomers. 2. Stereo-and enantioselective degradation in sewage sludge and soil. Environ Sci Technol 1995;29:2031-2037.

17. Fisher D, Hayes AL. A comparison of the biochemical and physiological effects of the systemic fungicide cyprofuram with those of the related compounds metalaxyl and metolachlor. Crop Protect 1985;4:501-510.

18. Nuninger C, Watson G, Leadbitter N, Ellgehausen H. CGA329351: introduction of the enantiomeric form of the fungicide metalaxyl. British Crop Protection Council, Farnham: United Kingdom; 1996.

19. Buerge IJ, Poiger T, Müller MD, Buser H-R. Enantioselective degradation of metalaxyl in soils: chiral preference changes with soil pH. Environ Sci Technol 2003;37:2668-2674.

20. Buser H-R, Müller MD, Poiger T, Balmer ME. Environmental behavior of the chiral acetamide pesticide metalaxyl: enantioselective degradation and chiral stability in soil. Environ Sci Technol 2002;36:221-226.

21. Jarman JL, Jones WJ, Howell LA, Garrison AW. Application of capillary electrophoresis to study the enantioselective transformation of five chiral pesticides in aerobic soil slurries. J Agric Food Chem 2005;53:6175-6182.

22. Monkiedje A, Zuehlke S, Maniepi SJN, Spiteller M. Elimination of racemic and enantioenriched metalaxyl based fungicides under tropical conditions in the field. Chemosphere 2007;69:655-663.

23. Qiu J, Wang Q, Wang P, Jia G, Li J, Zhou Z. Enantioselective degradation kinetics of metalaxyl in rabbits. Pest Biochem Physiol 2005;83:1-8.

24. Xu P, Diao J, Liu D, Zhou Z. Enantioselective bioaccumulation and toxic effects of metalaxyl in earthworm Eisenia foetida. Chemosphere 2011;83:1074-1079.

25. Zadra C, Marucchini C, Zazzerini A. Behavior of metalaxyl and its pure R-enantiomer in sunflower plants (Helianthus annus). J Agric Food Chem 2002;50:5373-5377.

26. Chapman AD. Numbers of living species in Australia and the world, 2009.

27. Wiegert RG, Evans FC. Investigations of secondary productivity in grasslands. In Secondary Productivity of Terrestrial Ecosystems: principles and methods Petrusewica K. Ed. Panstwowe Wydawnictwo Naukowe, Warszawa. Poland. 1967.

28. Kostaropoulos I, Papadopoulos AI, Metaxakis A, Boukouvala E, Papadopoulou-Mourkidou E. The role of glutathione S-transferases in the detoxification of some organophosphorus insecticides in larvae and pupae of the yellow mealworm, Tenebrio molitor (Coleoptera: Tenebrionidae). Pest Manage Sci 2001;57:501-508.

29. Prabhakar S, Chen MS, Elpidina E, Vinokurov K, Smith C, Marshall J, Oppert B. Sequence analysis and molecular characterization of larval midgut cDNA transcripts encoding peptidases from the yellow mealworm, Tenebrio molitor L. Insect Mol Biol 2007;16:455-468.

30. Effenberger F, Burkard U, Willfahrt J. Aminosäuren, 4. Enantioselektive Synthese $\mathrm{N}$-substituierter $\alpha$-Aminocarbonsäuren aus $\alpha$-Hydroxycarbonsäuren. Liebigs Ann Chem 1986;1986:314-333.

31. Palla O, Mirenna L, Colombo L, Zini G, Filippini L, Zanardi G. Fungicidal compositions based on (N-phenylacetyl-N-2, 6-XYLYL) methyl alaninate. Google Patents; 2001.

32. Elliel E, Wilen S, Mander L. Stereochemistry of organic compounds. Stereochemistry of Organic Compounds. Wiley: New York; 1994.

33. Testa B, Carrupt PA, Gal J. The so-called "interconversion" of stereoisomeric drugs: An attempt at clarification. Chirality 1993;5:105-111.

34. Hutt AJ, Caldwell J. The metabolic chiral inversion of 2-arylpropionic acids -a novel route with pharmacological consequences. J Pharm Pharmacol 1983;35:693-704. 
35. Friedman M. Chemistry, nutrition, and microbiology of D-amino acids. J Agric Food Chem 1999;47:3457-3479.

36. Tarrier N, Pilgrim H, Sommerfield C, Faragher B, Reynolds M, Graham E, Barrowclough C. A randomized trial of cognitive therapy and imaginal exposure in the treatment of chronic posttraumatic stress disorder. J Consult Clin Psychol 1999;67:13.
37. Gevao B, Mordaunt C, Semple KT, Piearce TG, Jones KC. Bioavailability of nonextractable (bound) pesticide residues to earthworms. Environ Sci Technol 2001;35:501-507.

38. Buckman AH, Brown SB, Hoekstra PF, Fisk AT. Toxicokinetics of three polychlorinated biphenyl technical mixtures in rainbow trout (Oncorhynchus mykiss). Environ Toxicol Chem 2004;23:1725-1736. 